\title{
Nasal Carriage, Risk Factors and Antimicrobial Susceptibility Pattern of Methicillin Resistant Staphylococcus aureus among School Children in Ethiopia
}

\author{
Alemayehu Reta ${ }^{1 *}$, Lealem Gedefaw ${ }^{2}$, Tsegaye Sewunet ${ }^{2}$ and Getenet Beyene ${ }^{2}$ \\ ${ }^{1}$ School of Medicine, College of Public Health and Medical Sciences, Debre Markos University, Ethiopia \\ ${ }^{2}$ Department of Medical Laboratory Science and Pathology, College of Public Health and Medical Sciences, Jimma, University, Ethiopia
}

\begin{abstract}
Background: Young children are major reservoir for community acquired Methicillin-resistant Staphylococcus aureus (CA-MRSA) and accelerate transmission of CA-MRSA.

Objective: The aim of this study was to determine the nasal carriage and antimicrobial resistance patterns of MRSA isolates among school children in Bahir Dar town, Ethiopia.

Methods: A community based cross-sectional study was conducted to determine the nasal carriage rate and antimicrobial susceptibility pattern of MRSA isolates among school children. A total of 300 nasal swabs were collected from March 1 to June 30, 2013. MRSA was detected using both Cefoxitin $(30 \mu \mathrm{g})$ and Oxacillin $(1 \mu \mathrm{g})$ discs in combination and risk factors were assessed using self-administered structured questionnaires. Statistical analysis
\end{abstract} was done using SPSS V-20.

Result: Of $123 \mathrm{~S}$. aureus isolates $17(13.8 \%)$ were MRSA isolates. The main risk factors for nasal carriage of MRSA in the study area were, having recurrent acute otitis media and use of an antibiotic in the previous year. The Susceptibility profiles of MRSA isolates were $(94.1 \%)$ to Chloramphenicol, Ciprofloxacin and Clindamycin, $(88.2 \%)$ to Ceftriaxone, Erythromycin and Trimethoprim-sulphamethoxazole and $(58.8 \%)$ to Doxycycline. All the isolates were resistant to Penicillin $G$ and sensitive to Gentamycin.

Conclusion: This study showed a rising rate of nasal carriage of MRSA among school children. Previous use of antibiotics by the children was statistically associated with MRSA carriage. Therefore developing decolonization protocols and proper utilization of drugs are needed in order to reduce the transmission and the burden of MRSA.

\section{Keywords:}

MRSA; Nasal carriage; Antibiotic resistance; School children

\section{Introduction}

Anterior nares are the most consistent site of colonization for Staphylococcus aureus. Carriage of S. aureus, including MRSA), is common in children, and genetic evidence suggests a relationship between nasal carriage of $S$. aureus and MRSA and subsequent invasive staphylococcal infection [1].

The carriage of CA-MRSA was significantly higher among children than among adults [2]. Moreover, living with young children was associated with increased risk of MRSA colonization in adults [3]. This shows that, young children were the major reservoir of MRSA in the community and were the main population responsible for the accelerated transmission of CA-MRSA and colonization with MRSA has a $25 \%$ probability of acquiring the infection than methicillinsensitive Staphylococcus aureus (MSSA) [2,3].

There has been a remarkable increase in the occurrence of $S$. aureus infections in general and CA- MRSA infections in particular [4]. Treatment of serious $S$. aureus infections can be challenging, and the associated mortality rate, among children, remains $20-25 \%$ despite the availability of highly active antimicrobial agents. However, most antistaphylococcal agents are ineffective against MRSA [5].

The spread of MRSA colonization occur especially in close contact areas like schools, pre-schools or households probably by the contaminated hands and surfaces [6].
The majority of afflicted children do not have identifiable risk factors for MRSA colonization. While some studies have demonstrated socioeconomic status, frequent exposure to antibiotics, healthcare worker in the home, child-care attendance, hospitalization, chronic illness and previous isolation of MRSA but there is no definite consensus as to why healthy children acquire these resistant bacteria [7-10].

MRSA is growing throughout the world and its prevalence ranges from $5-73 \%$. Across the globe, it was found to be the most common cause of bacteremia, respiratory, and skin infections $[11,12]$.

The African data on $S$. aureus, particularly on antibiotic susceptibility, are extremely limited [13]. The prevalence in Africa ranged from 5-45\% [14].

*Corresponding author: Alemayehu Reta, School of Medicine, College of Public Health and Medical Sciences, Debre Markos University, Ethiopia, Tel: 251587780844; E-mail: alemayehureta@yahoo.com

Received November 21, 2014; Accepted February 20, 2015; Published February 22, 2015

Citation: Reta A, Gedefaw L, Sewunet T, Beyene G (2015) Nasal Carriage Risk Factors and Antimicrobial Susceptibility Pattern of Methicillin Resistan Staphylococcus aureus among School Children in Ethiopia. J Med Microb Diagn 4 : 177. doi:10.4172/21610703.1000177

Copyright: (c) 2014 Reta A, et al. This is an open-access article distributed under the terms of the Creative Commons Attribution License, which permits unrestricted use, distribution, and reproduction in any medium, provided the original author and source are credited. 
Citation: Reta A, Gedefaw L, Sewunet T, Beyene G (2015) Nasal Carriage, Risk Factors and Antimicrobial Susceptibility Pattern of Methicillin Resistant Staphylococcus aureus among School Children in Ethiopia. J Med Microb Diagn 4: 177. doi:10.4172/21610703.1000177

In Ethiopia, MRSA is increasingly reported in hospitals starting from the first report since in 1987 [15]. According to Geyid at al., the isolation rate of MRSA was 30\% [15]. After two years (1993) a study by Tenssay et al. in Addis Ababa, $40 \%$ of the S. aureus isolates were found to be MRSA [16]. In another study at Jimma University Specialized Hospital, MRSA isolation rate increased to $51.8 \%$ [17]. Gabriel et al. found that MRSA isolation rate was $42.8 \%$ among healthy workers of Jimma specialized Hospital [18] and more recently, a study conducted in Jimma, indicates that $18.3 \%$ of school children's anterior nares were colonized by MRSA [19].

Accurate detection of Methicillin resistance in S. aureus is of the greatest importance to ensure effective treatment for the affected patient and to prevent further transmission. To our best knowledge, in Ethiopia most of studies on prevalence of MRSA are hospital based, and there is only a single study on isolation of MRSA from healthy school children. Hence this research will be an additional input and aimed at defining the current prevalence of MRSA nasal carriage among school children in Bahir Dar town, to determine the antimicrobial susceptibility pattern of the isolates, and to identify epidemiological risk factors associated with MRSA colonization.

\section{Materials and Methods}

A community based cross sectional study was conducted at nine primary schools from March1 to June 30 in Bahir Dar town. A total of 300 participants were recruited for this study. Multistage sampling technique was employed to randomly select primary school children at school type, grade and section level in order to collect the data and bacteriological samples. Individual variables, socio-demographic characteristics of the families and potential risk factors for MRSA nasal carriage were obtained from the children's guardians/parents immediately after written consent was obtained from the guardians/ parents plus verbal assent from the children (age greater than 7 years) before swabs were collected using self-administered pre-tested structured questionnaires prepared for this purpose.

\section{Specimen collection and processing}

Sterilized cotton swab was introduced $2-3 \mathrm{~cm}$ in the nasal cavity and rotated 4-5 times both clockwise and anticlockwise before withdrawal. A specimen was obtained from each child from both anterior nares and placed into Screw capped test tubes containing enrichment broth (contained $37.5 \mathrm{gm}$ sodium chloride $(\mathrm{NaCl}), 1.25$ gm yeast extract, 5.0 gm tryptone, and $500 \mathrm{ml}$ distilled water) [20] and the samples were labeled, packaged and transported to Bahir Dar regional laboratory within 2 hrs of collection and processed immediately according to standard procedures.

\section{Identification of S.aureus and MRSA}

Each $10 \mu$ of overnight incubated enrichment broth was inoculated in both blood agar and Mannitol Salt Agar (MSA) (Oxoid Ltd., England) and incubated at $37^{\circ} \mathrm{C}$ for $24-48 \mathrm{~h} \mathrm{[21]}$. Golden yellow colonies in the MSA and white creamy colonies in blood agar indicate $S$. aureus, which is subsequently identified by gram staining, catalase test, tube coagulase test and DNase test [22]. S. aureus isolates were tested for methicillin resistance by using modified Kirby-Bauer disc diffusion technique [23]. Inocula were adjusted at $0.5 \% \mathrm{McF}$ arland standard and each streaked uniformly with swab sticks in Mueller-Hinton agar plates containing $4 \% \mathrm{NaCl}$ to obtain confluent growth. The plates were allowed to dry for 3-5 minutes before the disks used. The Oxacillin $(1 \mu \mathrm{g})$ and Cefoxitin $(30 \mu \mathrm{g})$ discs were used for susceptibility testing. The plates were then incubated aerobically at $35^{\circ} \mathrm{C}$ for $24 \mathrm{hrs}$ [11]. The results were interpreted according to Clinical and Laboratory Standard Institute (CLSI) guidelines for Oxacillin and Cefoxitin susceptibility testing: a zone size of $\leq 10 \mathrm{~mm}$ was considered resistant; a zone size of $\geq 13$ $\mathrm{mm}$ was considered susceptible for Oxacillin and a zone size of $\leq 21$ $\mathrm{mm}$ was considered resistant; a zone size of $\geq 22 \mathrm{~mm}$ was considered susceptible for Cefoxitin [24].

\section{Antibiotic susceptibility testing}

The antibiotic susceptibility testing was performed by modified Kirby-Bauer disc diffusion technique. The colonies were picked up with wooden applicator stick and dipped into nutrient broth to make direct colony suspension of the isolates and inocula were adjusted at $0.5 \%$ McFarland standard. After few minutes these suspensions were streaked onto Mueller Hinton agar plates [23]. The antibiotic susceptibility testing was performed on the following antibiotic discs; Ceftriaxone (CRO) $(30 \mu \mathrm{g})$, Chloramphenicol (C) $(30 \mu \mathrm{g})$, Ciprofloxacin (CIP) $(5 \mu \mathrm{g})$, Clindamycin (DA) $(2 \mu \mathrm{g})$, Erythromycin (E) $(15 \mu \mathrm{g})$, Gentamicin $(\mathrm{CN})(10 \mu \mathrm{g})$, Penicillin G (P) (10 units), Trimethoprim-sulphamethoxazole (SXT) $(25 \mu \mathrm{g})$ and Vancomycin $(30$ $\mu \mathrm{g})$. All antibiotic discs/drugs were obtained from Oxoid Ltd. England. The plates were incubated at $37^{\circ} \mathrm{C}$ for $18-24 \mathrm{hrs}$ and interpretation made based on CLSI guidelines. S. aureus (ATCC-25923) was used as control for the antimicrobial susceptibility pattern.

\section{Data analysis}

The collected data was checked for completeness, coded, and then entered into Epi data version 3.1 software for edition and cleaning of data and export to SPSS version 20 Software for analysis. Univariate analysis such as, frequency distribution and percentages was performed. Binary and multiple logistic regressions analysis were used to check the presence of statistical significance association between independent and dependent variable by odds ratio (with $95 \%$ confidence interval). A p-value of $\leq 0.05$ was considered to be statistically significant. Finally data was organized and presented using tables and charts.

\section{Ethical consideration}

Ethical clearance was obtained from Jimma University ethical review board. Letter of permission to conduct the study was obtained from the director of each school. Informed consent was obtained from each study participant's guardian/parent and verbal assent from students. The purpose of the study was clearly described to the study participants including the benefits and risks of the study. Any information concerning the participants was kept confidential and the specimen collected from the participants was only analyzed for the intended purposes. The participants were enrolled only after they were sufficiently counseled and their informed consent has been obtained. Relevant confidentiality was maintained for every participant. After analysis of the participant's specimen, the positive findings for MRSA were decolonized by using $2 \%$ Muprocin which was given for 5 days (three times per day).

\section{Results}

\section{Socio demographic characteristics of study subjects}

A total of 300 study participants were involved in this study. The age of the studied children ranged from 6-12 years with mean age of $9.6+1.6$ years. About $52.3 \%(157 / 300)$ of the school children involved in the study were aged between $10-12$ years, and $51.0 \%(153 / 300)$ were females (Table 1). Most of the children's mother has high school educational status $33.0 \%$ (99/300), others $16.3 \%$ (49/300), $28.0 \%$ 
Citation: Reta A, Gedefaw L, Sewunet T, Beyene G (2015) Nasal Carriage, Risk Factors and Antimicrobial Susceptibility Pattern of Methicillin Resistant Staphylococcus aureus among School Children in Ethiopia. J Med Microb Diagn 4: 177. doi:10.4172/21610703.1000177

Page 3 of 6

\begin{tabular}{|c|c|c|c|c|c|c|}
\hline A & $20(57.1)$ & $15(42.9)$ & $35(11.7 \%)$ & $18(51.4)$ & $17(48.6)$ & $35(11.7 \%)$ \\
\hline B & $19(44.2)$ & $24(55.8)$ & $43(14.3)$ & $24(55.8)$ & $19(44.2)$ & $43(14.3)$ \\
\hline C & $17(37.0)$ & $29(63.0)$ & $46(15.3 \%)$ & $28(60.9)$ & 18(39.1) & $46(15.3 \%)$ \\
\hline D & $18(47.4)$ & $20(52.6)$ & $38(12.7 \%)$ & $14(36.8)$ & $24(63.2)$ & $38(12.7 \%)$ \\
\hline $\mathbf{E}$ & $10(55.6)$ & $8(44.4)$ & $18(6.0 \%)$ & $4(22.2)$ & $14(77.8)$ & $18(6.0 \%)$ \\
\hline $\mathbf{F}$ & $22(52.4)$ & $20(47.6)$ & $42(14.0 \%)$ & $22(52.4)$ & $20(47.6)$ & $42(14.0 \%)$ \\
\hline G & $18(46.2)$ & $21(53.8)$ & $39(13.0 \%)$ & $23(59.0)$ & $16(41.0)$ & $39(13.0 \%)$ \\
\hline $\mathbf{H}$ & $12(63.2)$ & $7(36.8)$ & $19(6.3 \%)$ & $11(57.9)$ & $8(42.1)$ & $19(6.3 \%)$ \\
\hline I & $7(35.0)$ & $13(65.0)$ & $20(6.7 \%)$ & $9(45.0)$ & $11(55.0)$ & $20(6.7 \%)$ \\
\hline Total & $143(47.7)$ & $157(52.3)$ & $300(100 \%)$ & $153(51.0)$ & $147(49.0)$ & $300(100 \%)$ \\
\hline
\end{tabular}

Table 1: Age and sex distribution of school children participated in the study in each school, Bahir Dar town, 2013.

(84/300), 22.7\% (68/300) were illiterate, elementary, college educational status respectively.

\section{Prevalence of MRSA}

From the total of 123 S. aureus isolate, 17(13.8\%) were found resistant to Methicillin/ Cefoxitin/Oxacillin. The prevalence of MRSA among primary school children was $13.8 \%(n=17)$. Slightly there was high percentage of MRSA (7.3\%) and S. aureus carriage (23.7\%) among school children between 10 and 12 years old. The prevalence of MRSA and S. aureus carriage among school children between 6 and 9 years old were $6.5 \%$ and $17.3 \%$ respectively. Of the total $17(13.8 \%)$ MRSA carriage, male accounts $12(9.8 \%)$ and female accounts 5(4.0\%). Like MRSA carriage, there was high carriage rate of $S$. aureus among male school children (71(23.7\%)) and the rest 52(17.3\%) were female school children (Tables 2 and 3 ).

\section{Evaluation of antibiotic susceptibility test}

A total of 17 MRSA isolates were subjected to antibiotic susceptibility test against 10 antimicrobial drugs. The susceptibility patterns of the isolates to commonly used antibiotics were, $88.2 \%$ to Ceftriaxone, Erythromycin and Trimethoprim-sulphamethoxazole, $94.1 \%$ to each of Chloramphenicol, Ciprofloxacin and Clindamycin, $100 \%$ to Gentamycin and $58.8 \%$ to Doxycycline. All the isolates were resistant to Penicillin G (Table 4). For all of the 17 MRSA isolates, the diameter of Vancomycin disk under disk diffusion test is between $15 \mathrm{~mm}$ and $19 \mathrm{~mm}$.

\section{Drug resistance pattern/antibiogram of MRSA}

The resistance patterns of MRSA isolates varied from 2 to 5 drugs and there was no MDR isolates (resistant to four or more drugs in addition to Cefoxitin/Oxacillin). The highest resistance pattern (47.06\%) was observed for three antibiotics with pattern of Penicillin/ Oxacillin/Cefoxitin. Two resistance patterns of equal proportion were observed for resistance to two and four antibiotics such as Penicillin/ Cefoxitin and Penicillin/Trimethoprim sulfamethoxazole/Oxacillin/ Cefoxitin, respectively. Only one resistance pattern (Penicillin/ Oxacillin/Cefoxitin) was observed to three antibiotics. Maximum resistance (resistant to five drugs) was observed for one MRSA isolate with resistance pattern of Penicillin/ Trimethoprim-sulfamethoxazole/ Doxycycline/Oxacillin/Cefoxitin (Table 5).

\section{Discussion}

In the present study, the overall frequency of isolation of $S$. aureus from primary school children of Jimma town was $41 \%$ (123/300). In a related study in Pokhara Nepal, [10], it was reported an isolation rate of $32.6 \% \mathrm{~S}$. aureus from nasal swabs of primary school children aged 6-10 years and another study in Duzce Turkey, [27], reported an isolation rate of $37 \%$ from nasal swabs of primary school children aged
6-14 years. According to a study conducted in Nigeria, [14], the rate of isolation of S. aureus from school children was $18.3 \%$ and also a recent study in Jimma, [19] the isolation rate for S. aureus was $47.3 \%$ among school children aged 5-15 years. The variation in the isolation rate of $S$. aureus among different studies could be due to difference in isolation techniques. The first three studies did not use enrichment broth) and difference in geographical distribution and population characteristics.

In our study, the prevalence of MRSA among primary school children aged 6-12 years was $13.8 \%$ (17/123). The finding of this study was in agreement with, a study conducted in Jimma [19], Nigeria [14] and Taiwan [2]. Our rate of MRSA isolation (13.8\%) is lower than that of the study conducted in Pokhara, Nepal [10], where the isolation rate was $56.1 \%$. This increased isolation rate of MRSA could be due to differences in isolation technique (they used only Oxacillin disk to detect MRSA which is affected by test conditions and also the MullerHinton Agar do not contain $4 \% \mathrm{NaCl}$ ) and there might be specific risk factors which are not assessed by this study that might increase the isolation rate.

In the current study, nasal colonization of MRSA among primary school children could be accounted for by the associated risk factors. The main risk factors after performing multivariate analysis were having recurrent AOM (three episodes of AOM in 6 months or more than three episodes in 12 months), use of an antibiotic in the previous 12 month and use of an antibiotic by a child of the household in the previous 12 month. As clearly shown in the result section, MRSA nasal carriage was significantly associated to having recurrent AOM three episodes of AOM in 6 months or more than three episodes in 12 months. MRSA can be one of the causes of AOM [28], so if there is a frequent contact with their ear and nose without washing their hands, MRSA can easily entered to the nose and finally the nose could be colonized by MRSA and also there might be a possibility of internal dissemination of the bacteria. On the other hand, those children who have recurrent AOM may take antibiotics repeatedly that might lead to the development of resistant bacteria.

In this study, $15(88.2 \%)$ of MRSA strains were isolated from children that had history of use of an antibiotic in the previous 12 month and also 10(58.8\%) from children that had history of use of an antibiotic by a child of the household in the previous 12, both of them had a statistically significant association with MRSA nasal carriage of primary school children aged 6-12 years. In a related study conducted at school children in Taipei, Taiwan, [1] also reported that, there was a statistically significant association between MRSA nasal carriage of children aged $>5$ years and history of use of an antibiotic in the previous 12 month. Even though, history of previous hospitalization was not statistically significant in the current study, the above mentioned study shown a statistical association of previous year hospitalization 
Citation: Reta A, Gedefaw L, Sewunet T, Beyene G (2015) Nasal Carriage, Risk Factors and Antimicrobial Susceptibility Pattern of Methicillin Resistant Staphylococcus aureus among School Children in Ethiopia. J Med Microb Diagn 4: 177. doi:10.4172/21610703.1000177

Page 4 of 6

\begin{tabular}{|c|c|c|c|c|c|}
\hline \multicolumn{2}{|c|}{ Study characteristics } & \multicolumn{2}{c|}{ MRSA carriage } & \multicolumn{2}{c|}{ S. aureus carriage } \\
\hline \multirow{2}{*}{ Age (year) } & & Yes & No & Nos & No $(\%)$ \\
\cline { 2 - 6 } & & No $(\%)$ & No $(\%)$ & $52(36.4)$ & $91(63.6)$ \\
\hline \multirow{3}{*}{ Sex } & $10-9$ & $9(12.7)$ & $44(84.6)$ & $71(45.2)$ & $86(54.8)$ \\
& Total & $8(15.4)$ & $62(87.3)$ & $123(41.0)$ & $177(59.0)$ \\
\cline { 2 - 6 } & Male & $17(13.8)$ & $106(86.2)$ & $71(46.4)$ & $82(53.6)$ \\
\hline & Female & $12(16.9)$ & $59(83.1)$ & $52(35.4)$ & $95(64.6)$ \\
\cline { 2 - 6 } & Total & $5(9.6)$ & $47(90.4)$ & $123(41.0)$ & $177(59.0)$ \\
\hline
\end{tabular}

Table 2: Age and sex distribution of MRSA and S. aureus among the school children, Bahir Dar town, 2013.

\begin{tabular}{|c|c|c|c|c|c|}
\hline \multirow{2}{*}{\multicolumn{2}{|c|}{ Risk factors }} & \multicolumn{4}{|c|}{ MRSA carriage } \\
\hline & & Yes (\%) & No (\%) & $\mathrm{P}$ & $\operatorname{AOR}(95 \% \mathrm{Cl})$ \\
\hline \multicolumn{6}{|c|}{ Child factors } \\
\hline \multirow{2}{*}{ Recurrent AOM } & Yes & $4(23.5)$ & $15(5.3)$ & 0.024 & $4.906(1.237-19.447)$ \\
\hline & No & $13(76.5)$ & $268(94.7)$ & 1 (ref) & 1 (ref) \\
\hline \multirow{2}{*}{ Use of antibiotic } & Yes & $15(88.2)$ & $144(50.9)$ & 0.042 & $5.063(1.062-24.145)$ \\
\hline & No & $2(11.8)$ & $139(49.1)$ & 1 (ref) & 1 (ref) \\
\hline \multirow{2}{*}{ Hospitalization } & Yes & $5(29.4)$ & $25(8.8)$ & 0.241 & $2.150(0.598-7.724)$ \\
\hline & No & $12(70.6)$ & $258(91.2)$ & 1 (ref) & 1 (ref) \\
\hline \multirow{2}{*}{ Sex } & Male & $12(70.6)$ & $59(55.7)$ & 0.237 & $1.991(0.635-6.236)$ \\
\hline & Female & $5(29.4)$ & $47(44.3)$ & 1 (ref) & 1 (ref) \\
\hline \multicolumn{6}{|c|}{ Household factor } \\
\hline \multirow{2}{*}{$\begin{array}{c}\text { Use of antibiotic by a child of the } \\
\text { household }\end{array}$} & Yes & $10(58.8)$ & $78(27.6)$ & 0.034 & $3.275(1.091-9.829)$ \\
\hline & No & $7(41.2)$ & $205(72.4)$ & 1 (ref) & 1 (ref) \\
\hline \multirow{2}{*}{ Hospitalization of family member } & Yes & $6(35.3)$ & $55(19.4)$ & 0.409 & $1.647(0.504-5.387)$ \\
\hline & No & $11(64.7)$ & $228(80.6)$ & 1 (ref) & 1 (ref) \\
\hline
\end{tabular}

Note: - AOM: Acute Otitis Media; ref: Reference; AOR: Adjusted Odds Ratio; Cl: Confidence Interval

Table 3: Multiple logistic regression analysis of risk factors for MRSA nasal carriage among primary school children, Bahir Dar town, 2013.

\begin{tabular}{|c|c|c|c|c|}
\hline \multirow{2}{*}{ Antimicrobial drugs } & \multicolumn{3}{|c|}{ MRSA isolates susceptibility pattern (No (\%)) } & \multirow[t]{2}{*}{ p-value } \\
\hline & $\mathrm{S}$ & 1 & $\mathrm{R}$ & \\
\hline Ceftriaxone & $15(88.2)$ & $2(11.8)$ & $0(0)$ & 0.016 \\
\hline Chloramphenicol & 16(94.1) & $1(5.9)$ & $0(0)$ & 0.001 \\
\hline Ciprofloxacin & $16(94.1)$ & $1(5.9)$ & $0(0)$ & 0.001 \\
\hline Clindamycin & $16(94.1)$ & $1(5.9)$ & $0(0$ & 0.001 \\
\hline Erythromycin & $15(88.2)$ & $2(11.8)$ & $0(0)$ & 0.016 \\
\hline Gentamycin & $17(100)$ & $0(0)$ & $0(0)$ & 0.000 \\
\hline Penicillin G & $0(0)$ & $0(0)$ & $17(100)$ & - \\
\hline $\begin{array}{c}\text { Trimethoprim- } \\
\text { sulphamethoxazole }\end{array}$ & 15(88.2) & $0(0)$ & $2(11.8)$ & 0.016 \\
\hline Doxycycline & $10(58.8)$ & $5(29.4)$ & $2(11.8)$ & 0.000 \\
\hline
\end{tabular}

Note: - S: Sensitive; I: Intermediate; R: Resistant

Table 4: Antibiotic susceptibility patterns of MRSA isolates, Bahir Dar town, 2013.

with nasal carriage of MRSA isolates among school children. Similarly, history of previous hospitalization of the family member is considered a risk factor for acquiring MRSA carriage however; in our study history of previous hospitalization of the family member was not identified as a statistically significant factor associated with MRSA nasal carriage.

Mother's schooling was not a factor significantly associated with MRSA nasal carriage in our study. However; a mother's high level of education has been demonstrated by other study [29] as independent protective factor for nasal carriage.

In general, there is no common consensus for risk factors of MRSA among different geographical area around the globe.

All MRSA isolates showed $100 \%$ resistance to Penicillin. This is in agreement with a study conducted in Nigeria, [14] where $100 \%$ resistance to Penicillin reported. Likewise, Abera et al. reported $100 \%$ resistance to Penicillin of MRSA strains isolated from clinical sources in Bahir Dar [30]. In addition, 100\% resistance to Penicillin of MRSA strains isolated from school children and prisoners reported in Jimma [19]. Significant proportions of MRSA isolates (88.2\%) were susceptible to Ceftriaxone, Chloramphenicol (94.1\%), Ciprofloxacin (94.1\%), Clindamycin (94.1\%), Erythromycin (88.2\%), Gentamycin (100\%), Trimethoprim-sulphamethoxazole (88.2\%) and Doxycycline (58.8\%). Comparable to our result, a study conducted in Pakistan reported that, $93 \%$ of MRSA isolates were sensitive to Chloramphenicol [31]. A study in Pokhara, Nepal showed that, $90.6 \%, 84.4 \%$ and $93.7 \%$ of MRSA isolates were sensitive to Erythromycin, Doxycycline and Ciprofloxacilin, respectively [10]. Similarly, a study conducted in North Carolina children revealed that, $98.6 \%$ of MRSA isolates were sensitive to Trimethoprim-sulphamethoxazole and $90.8 \%$ of MRSA isolates were sensitive to Clindamycin [7]. Differences in susceptibility pattern of the isolates could be due to differences in geographical area 
Citation: Reta A, Gedefaw L, Sewunet T, Beyene G (2015) Nasal Carriage, Risk Factors and Antimicrobial Susceptibility Pattern of Methicillin Resistant Staphylococcus aureus among School Children in Ethiopia. J Med Microb Diagn 4: 177. doi:10.4172/21610703.1000177

\begin{tabular}{|c|c|c|}
\hline No of antibiotics & Drug resistance pattern & Resistant strains (No (\%)) \\
\hline \multirow{2}{*}{$\mathbf{2}$} & Pen/Oxa & $4(23.53)$ \\
\hline \multirow{2}{*}{$\mathbf{3}$} & Pen/Fox & $2(11.76)$ \\
\hline \multirow{2}{*}{$\mathbf{4}$} & Pen/Oxa/Fox & $8(47.06)$ \\
\hline \multirow{2}{*}{$\mathbf{5}$} & Pen/SXT/Oxa/Fox & $1(5.88)$ \\
\cline { 2 - 3 } & Pen/Dox/Oxa/Fox & $1(5.88)$ \\
\hline
\end{tabular}

Note: - Pen: Penicillin G; SXT: Trimethoprim-Sulfamethoxazole; Fox: Cefoxitin Oxa: Oxacillin;

Dox: Doxycycline

Table 5: Drug resistance pattern of MRSA isolates for different antibiotics, Bahir Dar town, 2013

and the time where MRSA isolates first occurred (the longer the MRSA are present in a geographic area, the more likely they are to be resistant to other antibiotic classes). MRSA isolates were highly susceptible to most tested antibiotics, these could be due to CA-MRSA strains were capable of resisting only $\beta$ - lactam antibiotics as the result of carriage of genetic element SCCmec type IV. SCCmec type IV is one of the shorter SCCmec variations less likely to carry multi-drug resistance [9]. A few MRSA isolates showed resistance to the tested drug, these might be due to the possible mixing of HA-MRSA and CA-MRSA [32].

\section{Conclusion}

The overall prevalence of MRSA and S.aureus among primary school children was $13.8 \%$ and $41 \%$, respectively and the main predictors for nasal carriage of MRSA were, having recurrent AOM, use of an antibiotic in the previous 12 month, and use of an antibiotic by a child of the household in the previous 12 month. The risk factors investigated in our present work are responsible for increased rate of transmission of HA-MRSA from Hospital to the community. All MRSA isolates were resistant to Penicillin G and susceptible to Gentamycin and shown a reduced susceptibility to Doxycycline (58.8\%). Most of MRSA isolates were highly susceptible to the tested antibiotics. In general, based on the finding, there is a high prevalence of $S$. aureus and MRSA specifically, in school children and generally, in the community.

\section{References}

1. Lo WT, Wang CC, Lin WJ, Wang SR, Teng CS, et al. (2010) Changes in the nasal colonization with methicillin-resistant Staphylococcus aureus in children: 2004-2009. PLoS One 5: e15791.

2. Chen CJ, Hsu KH, Lin TY, Hwang KP, Chen PY, et al. (2011) Factors associated with nasal colonization of Methicillin-resistant Staphylococcus aureus among healthy children in Taiwan. J Clin Microbiol 49: 131-137.

3. Miller MB, Weber DJ, Goodrich JS (2011) Prevalence and risk factor analysis for Methicillin-resistant Staphylococcus aureus nasal colonization in children attending child care centers. J Clin Microbiol 49: 1041-1047.

4. Daum RS (2007) Clinical practice. Skin and soft-tissue infections caused by methicillin-resistant Staphylococcus aureus. N Engl J Med 357: 380-390.

5. Fridkin SK, Hageman JC, Morrison M, Sanza LT, Como-Sabetti K, et al. (2005) Methicillin-resistant Staphylococcus aureus disease in three communities. N Engl J Med 352: 1436-1444.

6. Peacock SJ, Justice A, Griffiths D, de Silva GD, Kantzanou MN, et al. (2003) Determinants of acquisition and carriage of Staphylococcus aureus in infancy. J Clin Microbiol 41: 5718-5725.

7. Shapiro A, Raman S, Johnson M, Piehl M (2009) Community-acquired MRSA infections in North Carolina children: prevalence, antibiotic sensitivities, and risk factors. N C Med J 70: 102-107.

8. Woods SE, Beiter E, Drake B, Engel A (2011) The prevalence of asymptomatic Methicillin-resistant Staphylococcus aureus in school-age children. E J Med 16: $18-20$.
9. Dietrich DW, Auld DB, Mermel LA (2004) Community-acquired methicillinresistant Staphylococcus aureus in southern New England children. Pediatrics 113: e347-352.

10. Rijal KR, Pahari N, Shrestha BK, Nepal AK, Paudel B, et al. (2008) Prevalence of methicillin resistant Staphylococcus aureus in school children of Pokhara. Nepal Med Coll J 10: 192-195.

11. Ankur B, Devjyoti M, Barnali P (2008) Prevalence of nasal carriage Methicillinresistant staphylococci in healthy population of Gangtok, East Sikkim. JIMSA 21: 191-193.

12. Diekema DJ, Pfaller MA, Schmitz FJ, Smayevsky J, Bell J, et al. (2001) SENTRY Participants Group: Survey of infections due to Staphylococcus species: frequency of occurrence and antimicrobial susceptibility of isolates collected in the United States, Canada, Latin America, Europe, and the Western Pacific region for the SENTRY Antimicrobial Surveillance Program. Clin Infect Dis 32:114-132.

13. Scragg JN, Appelbaum PC, Govender DA (1978) The spectrum of infection and sensitivity of organisms isolated from African and Indian children in a Durban hospital. Trans R Soc Trop Med Hyg 72: 325-328.

14. Okwu M, Bamgbala S, Aborisade W (2012) Prevalence of nasal carriage of community-associated Methicillin-resistant Staphylococcus aureus (CA-MRSA) among healthy primary school children in Okada, Nigeria. JNSR 2: 2224-3186.

15. Geyid A, Lemeneh $Y$ (1991) The incidence of methicillin resistant S. aureus strains in clinical specimens in relation to their beta-lactamase producing and multiple-drug resistance properties in Addis Abeba. Ethiop Med J 29: 149-161.

16. Tenssay Z, Tesfaye H (1993) Multiply resistant enteric pathogens in infant feeds, feeding teats and stools of bottle-fed babies from Addis Ababa. Bull of JIHS 3: 61-71.

17. Balta B, Derbie F (2003) Nasal Carriage of Methicillin Resistant S. aureus Ethiop J Health Sci 13: 108-109.

18. Gabriel R, Kebede E (2007) Nasal Carriage and Drug Sensitivity of Staphylococcus aureus among health workers of Jimma University Specialized Hospital, Southwestern Ethiopia. Ethiop J Health Sci 17: 73-79.

19. Kejela T, Bacha K (2013) Prevalence and antibiotic susceptibility pattern of Methicillin-resistant Staphylococcus aureus (MRSA) among primary school children and prisoners in Jimma Town, Southwest Ethiopia. ACMA 12:11.

20. Broekema NM, Van TT, Monson TA, Marshall SA, Warshauer DM (2009) Comparison of cefoxitin and oxacillin disk diffusion methods for detection of mecA-mediated resistance in Staphylococcus aureus in a large-scale study. J Clin Microbiol 47: 217-219.

21. Brown DF, Edwards DI, Hawkey PM (2005) Guidelines for the laboratory diagnosis and susceptibility testing of Methicillin-resistant Staphylococcus aureus (MRSA). J Antimicrob Chemother 56:1000-1008.

22. Kim YM, Oh CE, Kim SH, Lee J, Choi EH, et al. (2010) Nasal carriage of Staphylococcus aureus from healthy children attending day care center. Korean J Pediatr Infect Dis 17: 9-15.

23. Forbes BA, Sahm DF, Weissfeld AS (1998) Bailey \& Scott's Diagnostic Microbiology, Mosby Inc. USA.

24. Cheesbrough M (2002) District Laboratory Practice in Tropical Countries 2 Cambridge University Press, England.

25. Clinical and Laboratory Standards institute (2011) Performance standards for antimicrobial disc susceptibility tests. Twelfth International Supplement M100-S20.

26. Cabrera EC, Ramirez-Argamosa DT, Rodriguez RDM (2010) Prevalence of community acquired Methicillin-resistant Staphylococcus aureus from inmates of the Manila City Jail, characterization for SCCmec type and occurrence of Panton-Valentine leukocidin gene. Philipp Sci Letters 3:1-5.

27. Yildirim M, Sahin I, Basak S (2007) The investigation of nasal MRSA carriage and colonization of nasopharyngeal pathogens at a primary School in Duzce. Turk J Med Sci 37: 359.

28. Shawwa AB, Wegner D (2005) Trimethoprim-Sulfamethoxazole plus topical antibiotics as therapy for acute otitis media with otorrhea caused by Community-acquired Methicillin-Resistant Staphylococcus aureus in Children. Arch Otolaryngol Head Neck Surg 131:782-784.

29. Lamaro-Cardoso J, de Lencastre H, Kipnis A, Pimenta FC, Oliveira LS et al. (2009) Molecular epidemiology and risk factors for nasal carriage of 
Citation: Reta A, Gedefaw L, Sewunet T, Beyene G (2015) Nasal Carriage, Risk Factors and Antimicrobial Susceptibility Pattern of Methicillin Resistant Staphylococcus aureus among School Children in Ethiopia. J Med Microb Diagn 4: 177. doi:10.4172/21610703.1000177

Page 6 of 6

staphylococcus aureus and methicillin-resistant $S$, aureus in infants attending day care centers in Brazil. J Clin Microbiol 47: 3991-3997.

30. Abera B, Alem A, Bezabih B (2008) Methicillin-resistant strains of Staphylococcus aureus and coagulase-negative Staphylococcus from clinical isolates at Felege Hiwot Refferal Hospital, North West Ethiopia. Ethiop Med J 46: $149-154$
31. Kaleem F, Usman J, Hassan A, Omair M, Khalid A, et al. (2010) Sensitivity pattern of Methicillin resistant Staphylococcus aureus isolated from patients admitted in a tertiary care hospital of Pakistan. Iran J Microbiol 2: 141-143.

32. Klevens RM, Edwards JR, Tenover FC, McDonald LC, Horan T, et al. (2006) Changes in the epidemiology of methicillin-resistant Staphylococcus aureus in intensive care units in US hospitals, 1992-2003. Clin Infect Dis 42: 389-391. 